\title{
Factors in the Prevalence of Suicidal Ideation Among Bereaved Women in Rural Communities, Imo State, Nigeria
}

\author{
Ann Ukachi Madukwe*, Ngozi Nkwakaego Sydney-Agbor, Richards Ebireonwu Ebeh \\ Department of Psychology, Faculty of Social Sciences, Imo State University, Owerri, Nigeria \\ Email address: \\ annemichael2002@yahoo.com (A. U. Madukwe) \\ ${ }^{*}$ Corresponding author \\ To cite this article: \\ Ann Ukachi Madukwe, Ngozi Nkwakaego Sydney-Agbor, Richards Ebireonwu Ebeh. Factors in the Prevalence of Suicidal Ideation Among \\ Bereaved Women in Rural Communities, Imo State, Nigeria. American Journal of Psychiatry and Neuroscience. \\ Vol. 9, No. 3, 2021, pp. 118-125. doi: 10.11648/j.ajpn.20210903.18
}

Received: July 25, 2021; Accepted: August 26, 2021; Published: August 31, 2021

\begin{abstract}
This study investigated the prevalence of suicidal ideation and social support, number-of-children and age-atbereavement as predictors of suicidal ideation among bereaved women. Respondents were 474 women who lost their spouses at least 6 months before the study, selected from rural communities in the three senatorial zones of Imo State via purposive sampling technique. Two validated questionnaires were administered to assess suicidal ideation and perceived social support, while number-of-children and age-at-bereavement were measured as continuous variables among respondents. The study adopted a cross sectional survey design and the generalized linear model statistics was employed for analyses with SPSS vs 21. The findings of the study showed that suicidal ideation was significantly prevalent. Social support and age-at-bereavement were found positive and negative relationship with suicidal ideation, they were also significant predictors of suicidal ideation. Number-of-children was found to have a minimal positive relationship with suicidal ideation, it did not predict suicidal ideation. The study recommends that suicidal ideation interventions for widows should always involve adequate assessment of their social support type not just level, and how old they were at the time of bereavement. The establishment of a communitybased program of social support with accompanying suicide prevention counselling for widows was strongly recommended.
\end{abstract}

Keywords: Age, Bereaved, Community, Nigeria, Rural, Suicidal Ideation, Women

\section{Introduction}

Most empirical studies on suicide, suicide ideation, suicidal behaviour or attempt in Nigeria have been focused on adolescents and college students' populations [1-3], individuals with chronic health conditions [4, 5], and in relation to Boko Haram bombings [6, 7]. A few considered suicidal behaviours in the adult population [8,9]. In a systematic review Alabi et al., reported findings on suicide and suicidal behaviours in Nigeria dating back to 1957, that showed increasing prevalence of suicidal behaviour with majority being males of below 30years of age. Despite the many studies they reviewed, none was found that considered suicide or suicidal ideation among the bereaved (male or female) [10]. This strange observation is important especially since suicide has become a public health challenge and bereavement is usually linked to suicidal thoughts as one of major stressful life events. So, one wonders why little or no studies have focused on suicidal ideation among the bereaved especially women. The loss of one's spouse poses a huge challenge for women living in rural Igbo communities because generally, husbands serve as providers as well as protectors and ensure the women sense of belonging not just to the family but the society too. Hence, spousal bereavement is considered stressful, devastating, and traumatic [11]. When a partner (spouse) dies, the surviving partner loses some sense of belongingness and esteem, experience great emotional loneliness and perceived loss of support [12]; all these would require adjustment by the surviving partner. The cognitive motivation theory of mental incongruity explains that the behavioural disposition of an individual is dependent on relationships between standards (i.e., what one wants) and cognitions (what one gets- the actual experience), incongruity arises when there is an imbalance in this relationships (system). The incongruity is expected to be greater when factors that could influence the change (e.g., finance, shelter, 
food, social and emotional support) are less favourable [12]. Based on this theory, how the bereaved perceives the assistance or support received, the giver and degree of the gift in relation to their former experience with the deceased spouse and their current desires go a long way to determine whether the received support (tangible or intangible) would serve as a buffer or be able to mitigate the impact of bereavement and consequent suicide ideation.

Suicide ideation refers to thoughts of engaging in behaviour intended to end one's life [13]. According to Glenn and Nock "suicide ideation and attempts are relatively rare in childhood; the rate increases dramatically in adolescence and remains high throughout adulthood" [14] (p. 2). It is described here as a widow's tendency to think, feel or behave in a way that portrays the desire or need to die. It is likely that widows would have such suicidal ideas when they are overwhelmed with the burden so many responsibilities (e.g., child rearing), receive minimal family support, become stigmatized/ostracized by family or community, and when denied access to the late spouse's resources. It is possible that the amount of social support a widow receives can cushion the likelihood of having suicide ideas. Perceived social supports [15-17], and supportive social environments have been associated with decreased suicidal ideation or attempts. Thus, we argue that if widows perceive or receive sufficient support (both tangible and non-tangible support), the probability for suicidal ideations would reduce and better survival adjustment skills may be developed. However, findings of research on the role of social support in coping with loss experiences has remained inconclusive. Aside social support, the present research also considered age-at-bereavement and the numberof-children as possible buffers that will mitigate the occurrence of suicidal ideation among bereaved rural women. Unfortunately, no previous studies were found that considered the last two variables in relation to suicidal ideation in any population. Age-at-bereavement refers to the woman's age when her spouse died, while number-of-children was used to measure the number of off springs produced by the marriage before death occurred. This study therefore is geared towards unravelling the extent to which widows experience suicidal ideation, the possible buffering impacts of social support from family, friends or significant others, age-at-bereavement and number-of-children.

Oyetunji et al., in a content analysis of suicide in Nigeria reported a mean age of 33.66years for suicidal cases, with majority being males and marital conflict as one of the precipitating factors [18]. The authors explained that over $40 \%$ live in semi-urban areas. Ogbolu et al., in a report on the pattern of crisis call to a suicide telephone helpline service in Nigeria revealed a $68.1 \%$ prevalence in suicidal ideation and $12.8 \%$ previously attempted suicide rate [19]. They reported a mean age of 25.8 years, and a $53.2 \%$ majority of female callers.

Evan and Richard conducted a study on social support as a protective factor in suicide. Participants from the study came from the 2007 Adult Psychiatric Morbidity Survey (APMS), an English nationally representative sample conducted from October 2006 to December 2007, of residents over the age of
16. The participants in the sample were $56.8 \%$ female and ranged from 16 to 95 years of age. The ethnic origin of the sample was $89.6 \%$ white, 3.0 blacks, 3.8 South Asia and the rest were of another ethnicity. The finding showed that social support is associated with decreased likelihood of lifetime suicide behavior [20].

Similarly, Ariella, Paul and Annie studied the relationship between social support and suicidal risk behaviour in a national sample of ethnically diverse sexual minority women. 150 participants consisted of cis-gender women who identified as lesbian, bisexual, queer or other nonheterosexual identities were recruited through a national online survey. Participants were between the age of 18 to 66 $(M=31.6 ; S D=11.95)$. Regarding to sexual orientation, $38.7 \%$ identified as lesbian, $32.7 \%$ as bisexual, $28.6 \&$ as queer or "other". Using regression, social support from family and from significant other were both uniquely and inversely associated with past suicidal ideation, and social support from family was uniquely and inversely associated with lifetime suicide behaviour. The findings revealed that social support from family was a strong predictor of suicidal ideation and behavior [21].

Furthermore, Evan, John and Karen carried out a study on social support and positive events as protective factors in suicide. 379 participants were administered measures of social support, life events, depressive symptoms, and suicide ideation. The results indicated that; social support had a direct protective effect on suicide ideation, social support and positive events acted as individual buffers in the relationship between negative events and suicide ideation. The finding provided evidence that social support and positive events act as protective factors against suicidal behavior [22].

Sydney-Agbor, Ebeh and Madukwe carried out a study on the prevalence and predictors of psychache among widows in Imo State, Nigeria, with 472 widows aged 25 to 79 years. The researchers assessed social support, length of widowhood, age-at-husband's death and number of children as predictor of psychache. The result showed that psychache was significantly prevalent, and that social support, length of widowhood and age-at-husband's death had negative relationships with and were significant predictors of psychache. While number-of-children was found to also have a negative relationship with psychache but was not a significant predictor of same [23].

\subsection{Objectives}

The main aim of the study is to discover the extent of suicidal ideation among bereaved rural women. Based on the data gathered, the contributions of social support (family, friends and significant others), age-at-bereavement, and number-of-children in predicting suicidal ideation among bereaved rural women were also analyzed.

\subsection{Hypotheses}

1) There is a significant difference in the prevalence of suicide ideation among bereaved rural women in Imo 
State.

2) Social support significantly predicts suicidal ideation among bereaved rural women in Imo State.

3) Age-at-bereavement significantly predicts suicidal ideation among bereaved rural women in Imo State.

4) Number-of-children significantly predicts suicidal ideation among bereaved rural women in Imo State.

\section{Method}

\subsection{Participants}

The respondents in this study comprised 474 widows drawn from the 3 geopolitical zones in Imo State using stratified random sampling technique. Three local government areas were selected randomly from each of the geo-political zones, these were further represented by 3 communities. Quota sampling was used to select a minimum of 30 participants from each community located in the sub-urban areas of the State. The quota sampling was used at this point because some communities are more populated than the others. The respondents were aged between 25 and 98 years with a mean age of 60.70 and standard deviation of 11.91 .

Inclusion Criteria: Only widows who are permanently residing in the chosen communities, who are not remarried nor planning to remarry, who are not psychiatric patients before bereavement and who were present when the study was conducted were selected. Exclusion Criteria: Newly widowed women those whose husbands died less than six months by the time of this research; those diagnosed as psychiatric patients before the death of their husbands and those who cannot give valid information were excluded.

\subsection{Instruments}

Two instruments were used in this study. Namely: Multidimensional Scale of Perceived Social Support and Suicide Ideation Scale.

Multidimensional Scale of Perceived Social Support (MSPSS) is a 12- item likert inventory developed by [24]. It has seven response choices ranging from Very Strongly Disagree (1) to Very Strongly agree (7). Sample items include: 'There is a special person who is around when I am in need', 'I can talk about my problems with my family', 'My friends really try to help me'. The scale has three subscales: Significant Other Subscale (items 1, 2, 5, \& 10), Family Subscale (items 3, 4, 8, \& 11), and Friends Subscale (items $6,7,9, \& 12$ ). In this study, the total scale was employed. The mean score of the total scale is obtained by dividing the sum of all scores by 12 . Higher scores indicate greater perceived social support. Previous research has shown the MSPSS to have strong internal consistency $(\alpha=.88)$ for the overall scale and high subscale alphas $(0.85-0.91)$, in addition to good construct validity [24].

The second scale is Suicide Behaviours Questionnaire Revised (SBQ-R) by [25]. The SBQ-R is designed to identify individuals at risk of committing suicide and specific risk behaviours. It consists of four items, each targeting a different dimension of suicidality: frequency, closeness to attempt and suicidal ideation in the past and future. Responses are measured in Likert format of 6-points for items 1 and 4 and 5-points for items 2 and 3. A sample item is: "Have you ever thought about or attempted to kill yourself'? A respondent's SBQ-R score is gotten by summing up all the scores checked, and total scores range from 3 to 18. A score of 2 and above on item 1 indicates high risk of suicidality for adults, while an SBQ-R total score of 7 and above shows that the individual has increased risk of committing suicide. For the present study, during item analysis the items were reduced to three, eliminating item four with a loading of 0.22 . The three items yielded a Cronbach's alpha reliability of $0.88(\mathrm{~N}=144)$. Hence, only items 1, 2 and 3 were used in the study.

\subsection{Procedure}

Ethical approval for this study was gotten from the Faculty of Social Science, Imo State University, Owerri, Institution Based Research Review Board. The study started with an interaction with leaders of selected community (traditional heads and women leaders). This is in order to create familiarization and rapour with the leaders and gain their acceptance, assurance and support. An authorization letter from the ethical board was presented to the women leaders, who was also informed of the purpose of the research. Through these leaders, widows in each locality were contacted.

A cover letter (information sheet) introducing the researchers and the aim of the study and the consent form for signatures of those who agree to participate were attached to each questionnaire which was given to the participants. A total of 500 questionnaires were distributed, 50 per community, after explaining the purpose of the study to the participants. The researchers/research assistants aided the respondents to understand the questionnaire items where necessary. In each community, widows identified by the women leaders were assembled in the community town hall where the instruments were administered using a group approach. The questionnaires were expected to be completed within an hour of administration by each respondent, however, no time limits were given. The research process starting from first visit to community chiefs and women leaders lasted three days in each community. The participants were appreciated for their involvement in the research with a package of household items worth not more than five hundred naira ( $\$ 500.00)$ at the time of the study.

\subsection{Design and Statistics}

This is a descriptive study with cross sectional survey design. Data were analysed with the generalized linear binary logistic model of SPSS version 21. The interval data generated from suicidal ideation and social support scales were converted to ordinal data to enable binary logistics analysis. 
Table 1. Sociodemographic Characteristics of Respondents $(n=474)$.

\begin{tabular}{lll}
\hline & $\mathbf{n}$ & $\mathbf{\%}$ \\
\hline Educational Level & 101 & \\
None & 258 & 21.30 \\
Primary & 79 & 54.40 \\
Secondary & 36 & 16.70 \\
Tertiary & & 7.60 \\
Age at Bereavement & 150 & \\
$\leq 40$ years & 324 & 31.60 \\
$>40$ years & & 66.40 \\
Number of Children & 64 & \\
$\leq 2$ & 410 & 13.50 \\
$>2$ & & 86.50 \\
Resident Community & 52 & \\
Egbeada & 61 & 11.00 \\
Egbu & 20 & 12.90 \\
Nekede & 62 & 4.20 \\
Obiangwu & 48 & 13.10 \\
Obowu & 32 & 10.10 \\
Ogwa & 35 & 6.80 \\
Okwele & 59 & 7.40 \\
Orlu & 35 & 12.40 \\
Otulu & 70 & 7.40 \\
Ulakwo & & 14.80 \\
\hline
\end{tabular}

\begin{tabular}{lll}
\hline & n & \% \\
\hline Suicidal ideation- Score & & \\
Mild suicidal ideation & 397 & 83.80 \\
Severe suicidal ideation & 77 & 16.20 \\
\hline
\end{tabular}

\section{Result}

Table 1 above shows that respondents were mostly $(54.40 \%)$ primary school leavers, with only $7.60 \%$ who achieved tertiary level education. A $21.30 \%$ of no formal education was also found. The table also shows that $31.60 \%$ were bereaved on or before age forty (40 years), many $(86.50 \%)$ had more than two (2) children by the time they were bereaved. The least number of respondents $(4.20 \%)$ were from Nekede, while the majority $(14.80 \%)$ were from Ulakwo both communities are from the Owerri Senatorial zones. The result also shows that suicidal ideation was existent in these communities with $16.20 \%$ reporting severe suicidal ideation.

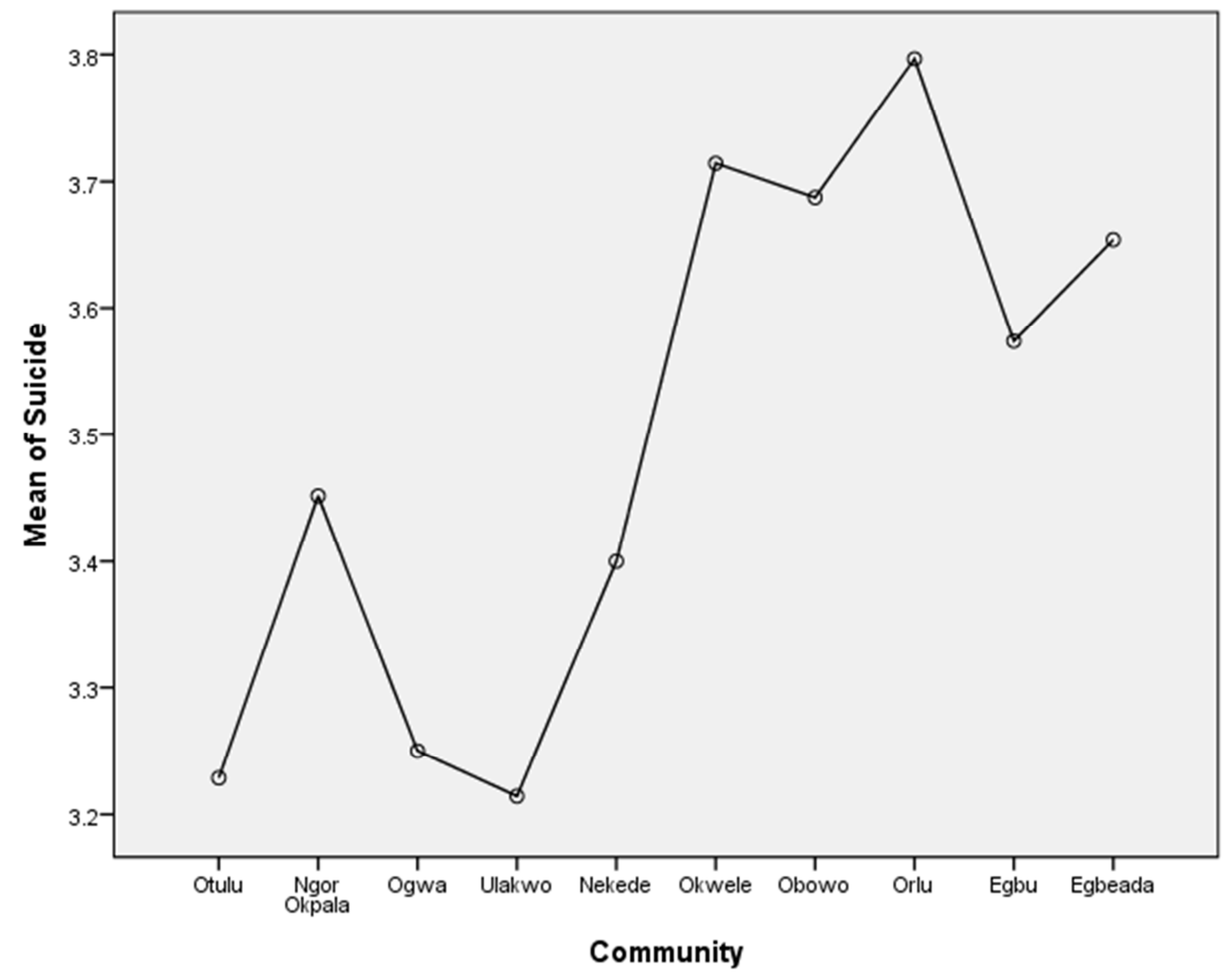

Figure 1. Showing mean plot of suicidal ideation by community.

Figure 1 above shows the rate of suicidal ideation across the communities studied. Suicidal ideation was least in Ulakwo with a mean score of (3.2) and most prevalent in the Orlu community with a mean score of (3.8). However, the provisions of the SBQ scoring guidelines states that scores below or equal to seven $(\leq 7)$ identifies at-risk individuals.
Table 2. Summary of Chi-Square Showing the prevalence of Suicidal Ideation.

\begin{tabular}{llll}
\hline & Observed N & Expected N & Residual \\
\hline 3 & 397 & 52.7 & 344.3 \\
4 & 17 & 52.7 & -35.7 \\
5 & 14 & 52.7 & -38.7 \\
6 & 18 & 52.7 & -34.7 \\
\hline
\end{tabular}




\begin{tabular}{llll}
\hline & Observed N & Expected N & Residual \\
\hline 7 & 11 & 52.7 & -41.7 \\
8 & 8 & 52.7 & -44.7 \\
9 & 6 & 52.7 & -46.7 \\
10 & 2 & 52.7 & -50.7 \\
11 & 1 & 52.7 & -51.7 \\
Total & 474 & & \\
\hline
\end{tabular}

$\chi^{2}(8)=2538.23, p<.001$
According to the result in Table 2 above the chi-square result $\left(\chi^{2}(8)=2538.23, \quad \mathrm{p}<.001\right) \quad$ indicates significant difference in the prevalence of suicidal ideation among bereaved women in Imo State rural communities. Most women reported low suicidal ideation. Hence, the first alternative hypothesis that there is a significant difference in the prevalence of suicidal ideation among bereaved women in Imo State rural communities was accepted.

Table 3. Summary of Multivariate Logistic Regression Analyses Showing a Predictor and Covariates of Suicidal Ideation.

\begin{tabular}{llllll}
\hline Parameters & B & SE & 95\% Wald CI & Wald $\boldsymbol{X}^{\mathbf{2}}$ & df \\
\hline (Intercept) & 1.79 & 0.07 & $1.65,1.94$ & 591.53 & 1 \\
Social Support (Low/High) & 0.103 & .03 & $.04, .17$ & 9.124 & 1 \\
Age-at-Bereavement & -.004 & 0.00 & $-.01,-.00$ & 12.272 & .000 \\
Number of Children & .001 & 0.01 & $-.01,-0.02$ & .034 & .003 \\
\hline
\end{tabular}

Note: Likelihood Ratio $\chi^{2}=25.45 ; \mathrm{p}<.001$

According to Table 3 above, the result $\beta=.10$ shows that social support level has a low positive relationship with suicidal ideation among bereaved rural women. For every increase in social support, suicidal ideation increases by 0.10 . This finding $\left(\chi^{2}(1)=9.12, \mathrm{p}<.05\right)$ was significant. Hence, the second alternative hypothesis that social support predicts suicidal ideation was accepted. Again, age-at-bereavement with regression coefficient $(\beta=-0.004)$ shows minimal negative relationship with suicidal ideation. The result $\left(\chi^{2}(1)=12.27, p<.001\right)$ was significant. Hence, the third alternative hypothesis that age-at-bereavement predicts suicidal ideation was accepted. Finally, number-of-children with regression coefficient $(\beta=0.001)$ shows minimal positive relationship with suicidal ideation. The result $\left(\chi^{2}(1)=.03\right.$, $\mathrm{p}>.05)$ was not significant. Hence, the fourth alternative hypothesis that number-of-children predicts suicidal ideation was rejected.

\section{Discussion}

The study investigated the prevalence of suicidal ideation in bereaved rural women and factors that contribute to it. Contributions of social support, age-at-bereavement and number-of-children at the time of widowhood were assessed in woman who lost their husbands at least six months before the commencement of the study. A significant difference in the prevalence of suicide ideation among bereaved rural women was found. Suicidal ideation varied across the communities and was found to be most prevalent among bereaved rural women from Orlu senatorial zone. Though, all the communities sampled in this study were of rural setting, some have more accommodating and supportive widowhood practices than others. This could explain the differences in prevalence rate. For instance, the Ulakwo community where suicidal ideation was least reported, have familial and community structures that ensure the bereaved woman receives social, emotional and financial support immediately the bereavement occurs. The community women organization (Ndom Aladinma) as well as the religious women organization (like the catholic women organization), each organize levels of visitations that commence with the announcement of bereavement. The visitation is done with food items, provision of water, and fellow women (in this case widows) who would cook and keep the bereaved company all day long. Hence, the bereaved rural woman has access to a social network that ensures that all her needs are met as adequately as possible during the early days and weeks of bereavement. This finding agrees with [14] assertion that suicidal ideation and attempts increases in adolescence and remains high in adulthood. All the women in the current study were either in late adolescence or adulthood when the bereavement occurred.

The results show that social support significantly predicts suicidal ideation, with a positive relationship. This finding contradicts that of Evan and Richard, who reported that social support is associated with decreased likelihood of lifetime suicide behaviours [20]; Sydney-Agbor, Ebeh and Madukwe that social support provides direct protective effect against suicidal ideation [23]. But the findings agree with Ariella et al., report that social support from the family and significant others was inversely associated with lifetime suicide behaviour and past suicidal ideation [21]. This finding brings to light the non-buffering role of perceived social support in suicidal ideation. Though many women reported receiving social support from their family and friends, most indicated that their hope lies in their significant other which is God (Jesus). These women were Christians of different denominations, each one referred to God (Jesus) as their ultimate support, and that their human relationships and support remain mostly unreliable. In many cases, the respondents view the support they receive from others as 'God's doing' and not necessarily a show of care or support by the helper or support giver. Therefore, many take to prayers as a way of getting support rather than indulging in any proactive social behaviour that could endear them to likely assistance. Hence, the finding that the higher the social support reported the greater the suicidal ideation reflects the respondents' belief that returning to God would solve all their problems; this is the apparent the local saying " $m$ 'anwu jee zuruike", which literally translates as "let me die and rest". This finding also reflects the position of the mental incongruity theory that how a person perceives social support 
in relation to their desires affects how they value the support. As such, if given social support is perceived as inadequate, then it will lose its power to mitigate against suicidal ideation. In addition, this finding mirrors the impact of the severe economic recession and consequent hardship being experienced by majority of Nigerians (both the givers and receivers of social support), which has resulted in increased reports of maladaptive behaviours (suicide) in the nation.

The third hypothesis was rejected because age-atbereavement was a significant predictor of suicidal ideation. The study revealed that the younger the age-at-bereavement the higher the suicidal ideation. This finding agrees with other studies in Nigeria that show the mean age of those involved in suicidal ideation, thoughts, attempts and suicide behaviour to be less than forty years. Oyetunji et al., reported a mean age of 33.66 years for suicide cases [18], while Ogbolu et al., reported a mean age of 25.8 years for suicide intervention callers [19]. Similarly, Sydney-Agbor, Ebeh and Madukwe reported a significant negative relationship between age-at-husband's death and psychache [23]. This study is unique because it considered bereavement across ages 15-65 years. Many of the women were bereaved around 40 years of age, hence, the bereavement was a major life stressor for them because it occurred in the prime of their youth. This youthful age could also be the major reason why the quality of perceived social support during bereavement is low. Family and friends may assume that the young bereaved woman does not need their help or support, hence, the seeming neglect or abandonment. Again, the young and beautiful looks of the recently bereaved woman, may engender fear (in potential male supporters) that the offered support could be misconstrued by the society, or in other cases hindered by existing cultural or traditional norms and practices which is most likely the case in the Orlu senatorial zone.

Finally, the fourth hypothesis was accepted because number-of-children was not a significant predictor of suicidal ideation. It is also best describes as having no association with suicidal ideation. This finding is similar to the report of Sydney-Agbor, Ebeh and Madukwe that non-significant negative relationship between number-of-children and psychache [23]. This finding is as expected since the presence of children at bereavement would be both a source of great comfort to the bereaved woman and a burden in light of child upbringing. In many cases, it is the worry over coping with child rearing responsibilities that causes the woman to need support. Still, the women consider these children as a major source of strength and reasons to hope for a better future.

\section{Implications}

For bereaved rural women this study has shown that suicidal ideation is a major concern. The commonness of the experience across all the communities reflects the need to address mental health concerns among these women as part of community health counselling. While addressing adjustment in bereavement, psychologists working with bereaved women should assess suicidal ideation and attempt as part of their protocol. The significant predictive ability of social support on increasing suicidal ideation points to the problem of extreme poverty in this population. Therefore, family members, community leaders, nongovernmental organizations, private sector individuals and the government interested in women affairs and the wellness of widows should first assess the actual needs of the women, push for the adequate implementation of policies that could address the needs (like the different poverty alleviation programs), then ensure that the benefits of such policies reach the grassroot population like women in rural communities, especially the young and widowed.

A major limitation of this work is that it focused only on widows, an assessment of suicidal ideation among widowers would have provided opportunity for a cross gender comparison, which may have given greater insights on the nature of suicidal ideation among widows.

\section{Conclusion}

The limitation notwithstanding, this study provides the needed scientific insights on the prevalence of suicidal ideation among bereaved rural women and the fact that when social support is less than expectation it fails to mitigate suicidality. The study therefore re-echoes the problem of social inequality and social exclusion in Nigeria. Policies and other measures used to provide support for bereaved rural women should make provisions that are as equivalent to the needs of the women as possible, for it to have the expected buffering or alleviating effect.

\section{Recommendations}

1. Periodic health needs assessment in rural communities of Imo State and Nigeria in general, that will prioritize the mental health status and well-being of the widows (women and girls) in the populace.

2. Equitable distribution of social and health resources by government and other stakeholders (especially the immediate community), is important to improve widows' perception of their overall well-being and inclusion in the society.

3. Psychologists in Nigeria must go beyond the classrooms and consultation rooms, to apply psychological science and principles in driving the message of health equity, social inclusion and social justice home in the larger society and to the Nigerian government in particular. Women and girls (widowed or not) should be able to get the support and services they require to achieve and sustain good mental well-being.

4. The study recommends that stringent laws aimed at protecting the right of widows and women in general be enacted. And that government, human rights activists and other relevant stakeholders must work together to ensure the effective implementation of existing laws as 
well as punishment of offenders. This will prevent the widows from suffering double losses (loss of spouse and loss of access to husband's properties $\}$ plus all other forms of exploitations, stigma, discrimination and abuse against them.

5. To reduce the increasing cases of several maladaptive behaviours for widows, women and girls in general, it is important to embrace more socially supportive and inclusive policies.

\section{Acknowledgements}

The authors acknowledge the financial support received from the Tertiary Education Trust Fund (TETFUND) Nigeria, to execute the project from which this article was developed. We also thank the management of Imo State University, Owerri, Nigeria for their role in securing the Institution based research grant and all other forms of support received from them.

\section{References}

[1] Adewuya, A. O., \& Oladipo, E. O. (2019). Prevalence and associated factors for suicidal behaviours (ideation, planning, and attempt) among high school adolescents in Lagos, Nigeria European Child \& Adolescent Psychiatry, 29, 1503-1512. doi.org/10.1007/s00787-019-01462-x.

[2] Ajibola, A. O. \& Agunbiade, O. M. (2021). Suicide ideation and its correlates among university undergraduates in south western Nigeria. International Quarterly of Community Health https://doi.org/10.1177/0272684X211004929.

[3] Ojuade, S. O., Munene, A., \& Mbutu, P. (2018). Severity of suicide behaviours among parasuicidal adolescents at federal neuropsychiatric hospital, Kappa-Lagos, Nigeria. African Journal of Clinical Psychology, 1 (2), 1-18.

[4] Agbir, T. M., Oyegeiya, M., Audu, M., Obindo, J., Goar, S., Piwuna, C., Obekpa, I., Nwoga, C., Armiyau, A. Y., Omidiji, N., \& Amedu, M. (2018). Suicide risk among psychiatric inpatients in North Central Nigeria. Jos Journal of Medicine, 12 (2), 24-32.

[5] Egbe, C. O., Dakum, P. S., Ekong, E., Kohrt, B. A, Minto, J. G. \& Ticao, C. J. (2017). Depression, suicidality, and alcohol use disorder among people living with HIV/AIDs in Nigeria. $B M C$ Public Health, 17 (542), 1-13.

[6] Ofodile, C., \& Nnatu, S. O. (2019). Child suicide bomber in the north east Nigeria: A victim or perpetrator? Nigerian Journal of Medical Sociology, 1 (1), 132-148.

[7] Olaniyan, A. (2017). Boko Haram insurgency and the spectre of female suicide bombers in Nigeria. An official conference proceeding of the IAFOR international conference on the social science held in Dubai, 1-14.

[8] Conejero, I., Olie, E., Courtet, P., \& Calati, R. (2018). Suicide in older adults: Current perspectives. Clinical Interventions in Ageing, 13, 691-699.

[9] Pengpid, S., \& Peltzer, K. (2020). The prevalence and correlates of suicidal ideation, plans and suicide attempts among 15-to 69-year-old persons in Eswatini. Behavioural Sciences, 10, 172-183. doi: 10.3390/bs10110172.

[10] Alabi, O. O., Alabi, A. I., Ayinde, O. O., \& Abdulmalik, J. O. (2015) suicide and suicidal behaviours in Nigeria: A review. Dokita, 5, 4-9.

[11] Oreh, C. I. (2006). Problems Associated with Widowhood. The Christian Mothers' Roles Toward Alleviating Them in The Mother's Voice. The Bulletin of The Catholic Women Organisation. St. Peter's Chaplaincy, University of Nigeria, Nsukka.

[12] Baarsen, B. V. (2002). Theories on coping with loss: The impact of social support and self-esteem on adjustment to emotional and social loneliness following a partner's death in later life. Journal of Gerontology, 7B (1), S33-S42.

[13] Nock, M. K., Borges, G., Bromet, E. J., Cha, C. B., Kessler, R. C., \& Lee, S. (2008). Suicide and suicidal behaviour. Epidemiology Review, 30 (1): 133-154.

[14] Glenn, C. R., \& Nock, M. K. (2014). Improving the short-term prediction of suicidal behaviour. Journal of Preventive Medicine, 47 (3), S176-S180.

[15] Mustanski, B., \& Liu, R. A. (2013). Longitudinal study of predictors of suicide attempts among lesbian, gay, bisexual, and transgender youth. Archives of Sexual Behaviour; 42 (3): 437-438.

[16] Liu, R., \& Mustanski, B. (2012). Suicidal ideation and selfharm in lesbian, gay, and transgender youth. American Journal of Preventive Medicine, 42 (3): 221-228.

[17] Reisner, S. L, Biello K, Perry N. S, \& Mimiaga, M. J. A (2014). Compensatory model of risk and resilience applied to adolescent sexual orientation disparities in non-suicidal selfinjury and suicide attempts. American Journal of Orthopsychiatry, 84 (5), 545-556.

[18] Oyetunji, T. P., Arafat, S. M. Y., Famori, S. O., Akinboyewa, T. B., Afolami, M., Moyo Faith Ajayi, M. F., \& Kar, S. K. (2021). Suicide in Nigeria: Observations from the content analysis of Newspaper. General Psychiatry, 34, e100347. doi: 10.1136/gpsych-2020-100347.

[19] Ogbolu, R. E., Oyatokun, B. O., Ogunsola, K., Quadri, O., Adegbite, T. A., Tade, T., Olafisoye, O., \& Aina, O. F. (2020). The pattern of crisis calls to a suicide telephone helpline service in Nigeria. Annals of Health Research, 6 (3), 246-257.

[20] Evan. M. K. \& Richard. T. L. (2013). Social Support as a Protective Factor in Suicide; Findings from two Nationally Representative Samples. Retrieved from www.ncbi.nlm.nih.gov/pmc/articles/PMC3683363/.

[21] Ariella. R. T., Paul. B. P., \& Annie. E. R. (2016). The Relationship between Social Support and Suicide Risk in National Sample of Ethnically Diverse Sexual Minority Women. Gay \& Lesbian Mental Health, 20 (2), 116-126. $\begin{array}{llll}\text { Retrieved } & \text { September } & 10 & \text { from }\end{array}$ https://www.ncbi.nlm.nih.gov/pmc/articles/ PMC5178968.

[22] Evan. M. K., John. H. R. \& Karen. E. S. (2013). Social Support and Positive Events as Suicide Resiliency Factors: Examination of Synergistic Buffering Effect. Retrieved from www.tandfonline.com/doi/full/10.1080/13811118.2013.82615 5 ? $\mathrm{src}=$ recsys. 
[23] Sydney-Agbor, N. N., Ebeh, R. E., \& Madukwe, A. U. (2021). Prevalence and predictors of psychache among widows in Imo State, Nigeria. Nigerian Journal of Social Psychology, 4 (1), 27-39.

[24] Zimet G. D, Dahlem N. W, Zimet S. G, \& Farley, G. K. (1988) The multidimensional scale of perceived social support. Journal of Personality Assessment, 52, 30-41.
[25] Osman, A., Bagge, C. L., Guitierrez, P. M., Konick, L. C., Kooper, B. A., \& Barrios, F. X. (2001). The suicidal behaviours questionnaire- revised (SBQ-R): Validation with clinical and nonclinical samples. Assessment, 5, 443-454. 